\title{
AVALIAÇÃO DE MODELO MATEMÁTICO DE PARÂMETROS DISTRIBUÍDOS APLICADO À SECAGEM DE GRÃOS DE UVA EM CAMADA FINA
}

\author{
G. JOHANN ${ }^{1 *}$, N.C. PEREIRA ${ }^{1}$, E.A. SILVA ${ }^{2}$ \\ ${ }^{1}$ Universidade Estadual de Maringá, Departamento de Engenharia Química \\ ${ }^{2}$ Universidade Estadual do Oeste do Paraná, Departamento de Engenharias e Ciências Exatas \\ "grajohann@yahoo.com.br
}

\begin{abstract}
RESUMO
Foi estudado o processo de secagem convectiva de grãos de uva em camada fina, proposto um modelo matemático de parâmetros distribuídos, um método de solução das equações obtidas e validados o modelo, método de resolução e as considerações por meio de comparação com dados da literatura. A modelagem matemática da secagem convectiva e as simplificações propostas, somadas ao método numérico utilizado para resolver o sistema de equações, foi eficiente na descrição do processo. Dentre as condições de secagem simuladas, os perfis de umidade experimental e calculado, apresentaram um erro médio de $2.3 \%$ e um erro máximo de $15 \%$. Na análise estatística do teste $\mathrm{F}$ e de $\mathrm{R}^{2}$, concluiu-se que o modelo obtido pode ser utilizado para fins preditivos. O coeficiente de transferência de massa, estimado pelo modelo, variou entre $3.32 \times 10^{-5} \mathrm{e}$ $2.15 \times 10^{-4} \mathrm{~m} \mathrm{~min}^{-1}$, condizentes com a literatura. Nos perfis de umidade e temperatura dos grãos em diferentes tempos de secagem, foi verificado que a transferência de massa é reduzida a medida que a secagem atinge os estágios finais, e que, partir do raio adimensional igual a 0.20 , existe a resistência à transferência de massa dentro dos grãos.
\end{abstract}

\section{INTRODUÇÃO}

De acordo com Menezes et al. (2014), a umidade média de grãos de uva é de aproximadamente $35 \%$ em base seca (b.s.). Para Baker (1997), o teor de umidade seguro para armazenamento de grãos varia de acordo com a espécie, girando em torno de 8 à $10 \%$ para oleaginosas e de 12 à 14\% para amiláceas. De modo que o menor teor de umidade torna viável o armazenamento por períodos superiores à um ano. Para Hellevang (2013), quanto maior o tempo de armazenamento, menor deverá ser o teor de umidade do grão. Ainda, de acordo com Baker (1997), caso a secagem seja conduzida na propriedade, em geral, aplicam-se altas temperaturas em regiões cuja umidade é elevada, caso contrário a secagem é conduzida em temperaturas mais amenas. Usualmente, os grãos que serão utilizados como semente não são expostos à temperaturas superiores a $50{ }^{\circ} \mathrm{C}$, já grãos cuja finalidade é o consumo após cozimento, ou a extração de óleo, são submetidos a temperaturas não superiores a $80{ }^{\circ} \mathrm{C}$ (Brooker et al. 1974).

Mais de 70 milhões de toneladas de água são removidas de grãos anualmente, para garantir o armazenamento adequado dos mesmos, isso é equivalente a evaporar um cubo de água com mais de $400 \mathrm{~m}$ de comprimento lateral (Raghavan e Sosle, 2007). Para Alonso (2011), a energia consumida na secagem de grãos é essencialmente empregada em três processos: aquecimento do grão; evaporação da água; e aquecimento do ar de 
secagem. Dessa forma, para que o processo seja eficiente, é necessário recuperar energia de um desses três processos, entretanto, o desenvolvimento dessas alternativas tem levado muitas décadas.

Nos processos de secagem, o estudo da cinética da secagem permite a predição da distribuição de temperatura e umidade no material e no equipamento, sendo essencial para o projeto e controle de processos, além de possibilitar a otimização. Assim, estudos de secagem conduzidos em convecção forçada, método de secagem mais amplamente utilizado, são essenciais para determinação das propriedades de transferência de calor e massa.

Por sua vez, os grãos de uva, subprodutos da indústria de suco de uva e correlatos, são usados principalmente para alimentação animal, porém recentemente vêm atraindo a atenção de pesquisadores devido aos componentes funcionais (Majd et al. 2013). Como, por exemplo, os ácidos graxos insaturados, que podem representar até cerca de $89 \%$ da composição total dos óleos (Davidov-Pardo e McClements, 2015). O processo de extração de óleo envolve diversas etapas preliminares, como limpeza; descasque; e secagem, sendo que esta última afeta diretamente a estrutura e qualidade dos produtos (Gutiérrez et al. 2008).

Diante do exposto, os objetivos do presente trabalho são propor um modelo matemático de parâmetros distribuídos, para descrever a secagem de grãos de uva em camada fina; propor um método de solução das equações obtidas; e validar o modelo, método de resolução e considerações por meio de comparação com dados da literatura.

\section{MATERIAIS E MÉTODOS}

\subsection{Modelagem do processo}

A modelagem do processo de secagem de grãos de uva em camada fina foi realizada com base em dados experimentais da literatura, Menezes (2014), cujo módulo experimental utilizado é representado na Figura 1, os grãos utilizados foram do cultivar Bordô e a secagem foi conduzida a temperaturas de 50,60, 70 e $80{ }^{\circ} \mathrm{C}$ e velocidade de $0.8,1.2$ e $1.6 \mathrm{~m} \mathrm{~s}^{-1}$.

Figura 1 - Módulo experimental utilizado por Menezes (2014)

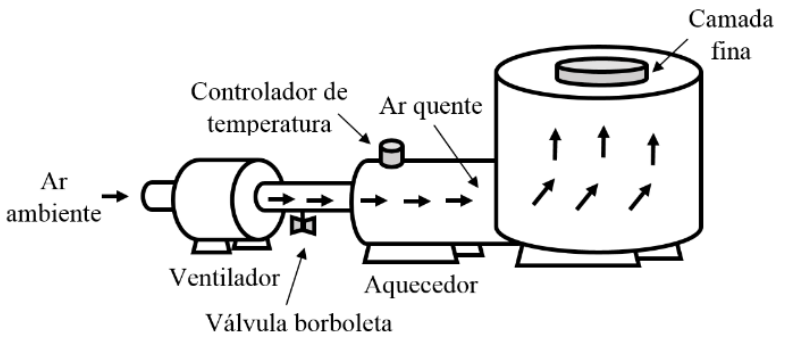

O módulo da Figura 1, foi constituído de um soprador de ar, com ajuste de velocidade realizado por um dumper, ligado a um duto metálico equipado com quatro resistências elétricas. A saída do duto metálico foi conectada à uma caixa de aço, por onde passava o ar aquecido, que segue até uma bandeja perfurada que serve de suporte para a amostra. A temperatura do ar de secagem foi ajustada a partir de resistências elétricas, controladas por um reostato. A indicação da temperatura do ar foi feita por um termopar, inserido logo abaixo da tela perfurada do secador.

$\mathrm{Na}$ solução de modelos difusionais, aplicados à secagem, em geral, são consideradas muitas suposições simplificadoras, como difusividade invariável com o teor de umidade, e resistência externa à transferência de massa desprezível, ou seja: a umidade na superfície do sólido instantaneamente alcança o valor de umidade de equilíbrio de grãos (Barrozo et al. 2006).

$\mathrm{Na}$ construção do presente modelo, foram consideradas as seguintes hipóteses simplificadoras: os grãos, aproximadamente esféricos, formam um leito fino; as perdas de calor pelas paredes do secador são desprezíveis; a umidade e temperatura iniciais das sementes são uniformes; transporte unidimensional de calor e massa nos grãos; 
distribuições uniformes de temperatura, umidade e velocidade do ar na entrada do secador; na transferência de calor entre o ar de secagem e os grãos, predomina o fenômeno da convecção; o ar de secagem comporta-se gás ideal, nas condições de modelagem; a difusão da umidade ocorre nos micro poros; as variações de temperatura e umidade no ar de secagem são desprezíveis.

Com base nessas considerações, foram efetuados balanços de massa e energia nos grãos, obtendo-se um conjunto de duas equações diferenciais parciais. Em coordenadas esféricas, com variação de umidade e temperatura radial, o balanço de massa em cada grão é dado pela Equação 1.

$\frac{\partial \mathrm{X}}{\partial \mathrm{t}}=\mathrm{D}_{\mathrm{eff}} \frac{\partial^{2} \mathrm{X}}{\partial \mathrm{r}^{2}}+\frac{2}{\mathrm{r}} \frac{\partial}{\partial \mathrm{r}}\left(\mathrm{D}_{\mathrm{eff}} \mathrm{X}\right)$

Em que $\mathrm{X}$ é a umidade do grãos em base seca (b.s.), t é o tempo (min), r é a coordenada radial $(\mathrm{m})$, e $\mathrm{D}_{\text {eff }}$ é o coeficiente de difusividade efetiva $\left(\mathrm{m}^{2} \min ^{1}\right)$.

Na secagem por convecção, a superfície do grão troca calor com o ambiente, como resultado da convecção e por condução interna, existindo, ainda, fluxo de energia saindo do grão devido à dessorção. Tem-se, portanto, o balanço de energia no grão, descrito pela Equação 2.

$\frac{\partial \mathrm{T}_{\mathrm{S}}}{\partial \mathrm{t}}=\propto_{\mathrm{S}} \frac{\partial^{2} \mathrm{~T}_{\mathrm{S}}}{\partial \mathrm{r}^{2}}+\propto_{\mathrm{S}} \frac{2}{\mathrm{r}} \frac{\partial \mathrm{T}_{\mathrm{S}}}{\partial \mathrm{r}}$

Em que $\mathrm{T}_{\mathrm{S}}$ é a temperatura do grão $\left({ }^{\circ} \mathrm{C}\right)$, e $\propto_{S}$ é a difusividade térmica do grão $\left(\mathrm{m}^{2} \min ^{-1}\right)$.

O processo de transferência de massa entre um sólido e um fluido é influenciado pela resistência interna e/ou externa ao transporte de massa. A resistência à transferência de massa interna depende principalmente das características do sólido e da temperatura (Simal et al. 1998), enquanto a resistência externa está ligada ao coeficiente de transferência de massa externa, relacionado à espessura da camada limite. Uma dessas resistências frequentemente prevalece, embora a verificação da importância relativa seja necessária para o estabelecimento das condições de processo (Rosselló et al. 1997). Como regra geral, a resistência à transferência de massa em sólidos é elevada, sendo possível estabelecer que uma característica geral da cinética do processo de transferência de massa é a sua relativa lentidão (Mullet et al. 2003).

Quando a resistência externa à transferência de massa é considerada negligenciável, a solução da Equação 1 pode ser obtida assumindo-se o equilíbrio termodinâmico, $\quad X(R, t)=X_{E}$. Porém, em alguns processos de secagem, a resistência externa não pode ser desconsiderada, devido principalmente à baixa velocidade do ar de secagem. Neste caso, a umidade na superfície do sólido é determinada considerando-se um coeficiente de transferência de massa entre o sólido e fluído (Simal et al. 2003).

No presente estudo, no instante imediatamente inicial à secagem, a umidade dos grãos foi considerada uniforme e igual à umidade inicial. No centro da esfera, considerou-se que não existia fluxo de umidade, por sua vez, na superfície da esfera, a massa que saía por difusão foi considerada igual à massa que entrava no ar de secagem. Assim, as condições inicial e de contorno foram representadas pelas Equações 3, 4 e 5 .

$$
\begin{aligned}
& \mathrm{t}=0, \mathrm{X}=\mathrm{X}_{0}, 0 \leq \mathrm{r} \leq \mathrm{R} \\
& \left.\frac{\partial \mathrm{X}}{\partial \mathrm{r}}\right|_{\mathrm{r}=0}=0 \\
& -\left.\frac{\partial}{\partial \mathrm{r}}\left(\mathrm{D}_{\text {eff }} \mathrm{X}\right)\right|_{\mathrm{r}=\mathrm{R}}=\mathrm{k}_{\mathrm{S}}\left(\mathrm{X}-\mathrm{X}_{\mathrm{E}}\right), \mathrm{t}>0
\end{aligned}
$$

Em que $\mathrm{X}_{0}$ é a umidade inicial (b.s.), $\mathrm{R}$ é o raio do grão $(\mathrm{m}), \mathrm{k}_{\mathrm{S}}$ é o coeficiente de transferência de massa $\left(\mathrm{m} \min ^{-1}\right)$, e $\mathrm{X}_{\mathrm{E}}$ é a umidade de equilíbrio (b.s.).

No instante imediatamente anterior aso início da secagem, a temperatura do grão é uniforme e igual à temperatura ambiente. No centro da esfera não existe fluxo de calor, por 
sua vez, na superfície da esfera, a energia que sai é igual à soma da energia que entra no ar de secagem devido à convecção e devido à evaporação. Assim, as condições inicial e de contorno são representadas pelas Equações 6, 7 e 8 .

$\mathrm{t}=0, \mathrm{~T}_{\mathrm{S}}=\mathrm{T}_{\mathrm{S}_{0}}, 0 \leq \mathrm{r} \leq \mathrm{R}$

$\left.\frac{\partial \mathrm{T}_{\mathrm{S}}}{\partial \mathrm{r}}\right|_{\mathrm{r}=0}=0$

$\left.\frac{\partial}{\partial \mathrm{r}}\left(\mathrm{\kappa}_{\mathrm{S}} \mathrm{T}_{\mathrm{S}}\right)\right|_{\mathrm{r}=\mathrm{R}}=\mathrm{h}_{\mathrm{c}}\left(\mathrm{T}_{\mathrm{G}}-\mathrm{T}_{\mathrm{S}}\right)-\mathrm{k}_{\mathrm{S}}(\mathrm{X}-$

$\left.\mathrm{X}_{\mathrm{E}}\right) \rho_{\mathrm{S}} \lambda_{\mathrm{D}}, \mathrm{t}>0$

Em que e $\mathrm{T}_{\mathrm{S}_{0}}$ é a temperatura inicial do grão $\left({ }^{\circ} \mathrm{C}\right), \kappa_{\mathrm{S}}$ é a condutividade térmica do grão (W $\mathrm{m}^{-1^{\circ}} \mathrm{C}^{-1}$ ), $\mathrm{h}_{\mathrm{c}}$ é o coeficiente de transferência de calor convectivo $\left(\mathrm{W} \mathrm{m}^{-1}{ }^{\circ} \mathrm{C}^{-1}\right)$, $\mathrm{T}_{\mathrm{G}}$ é a temperatura do ar de secagem $\left({ }^{\circ} \mathrm{C}\right), \lambda_{\mathrm{D}}$ é o calor de dessorção $\left(\mathrm{kJ} \mathrm{kg}^{-1}\right)$, e $\rho_{\mathrm{S}}$ é a densidade do grão $\left(\mathrm{kg} \mathrm{m}^{-3}\right)$.

$\mathrm{Na}$ resolução do sistema de equações diferenciais, Equações 1 e 2, foi empregado o método das Linhas, no qual as derivadas espaciais foram aproximadas por diferenças finitas, transformando o sistema de equações diferenciais parciais em um sistema de equações ordinárias. Para aplicar este método, primeiramente verificou-se que a divisão do domínio do problema, tanto em 10 como em 50 elementos de discretização, resultou em valores próximos (ordem de $10^{-8}$ ). Optou-se, portanto, em utilizar 10 elementos de discretização, de modo a reduzir o esforço computacional.

Para a resolução do sistema das equações, obtidas após a discretização das equações, foi empregado o software Maple $13^{\circledR}$, método numérico de Rosenbrook, por meio do comando dsolve, sendo que no problema de secagem investigado, a temperatura e a umidade inicial é a ambiente $\left(\mathrm{T}_{\mathrm{G}_{0}}, \mathrm{Y}_{0}\right)$, que repentinamente são aumentadas para $\mathrm{T}_{\mathrm{G}_{\text {feed }}}$ e $\mathrm{Y}_{\text {feed }}$, na alimentação do secador, e mantida neste valor. Esta condição de contorno foi modificada com o intuito de torná-la a contínua na variável tempo de acordo com as Equações 9 e 10.

$\mathrm{T}_{\mathrm{G}}=\mathrm{T}_{\mathrm{G}_{\text {feed }}}+\left(\mathrm{T}_{\mathrm{G}_{0}}-\mathrm{T}_{\mathrm{G}_{\mathrm{feed}}}\right) \mathrm{e}^{-\mathrm{st}}$

$\mathrm{Y}=\mathrm{Y}_{\text {feed }}+\left(\mathrm{Y}_{0}-\mathrm{Y}_{\text {feed }}\right) \mathrm{e}^{-\mathrm{st}}$

Em que $\mathrm{T}_{\mathrm{G}_{0}}$ é a temperatura ambiente do ar $\left({ }^{\circ} \mathrm{C}\right), \mathrm{T}_{\mathrm{G}_{\text {feed }}}$ é a temperatura de alimentação do ar de secagem $\left({ }^{\circ} \mathrm{C}\right)$, Y é a umidade do ar de secagem (b.s.), $Y_{\text {feed }}$ é a umidade de alimentação do ar de secagem (b.s.), $\mathrm{Y}_{0}$ é a umidade ambiente do ar (b.s.), e $\mathrm{s}=10^{5} \mathrm{~min}^{-1}$.

\subsection{Parâmetros do modelo}

As propriedades termo físicas dos grãos de uva, densidade e calor específico, foram determinadas em estudo anterior, Menezes (2014), sendo respectivamente $1260 \mathrm{~kg} \mathrm{~m}^{-3} \mathrm{e}$ $1.5 \mathrm{~kJ} \mathrm{~kg}^{-1}{ }^{\circ} \mathrm{C}^{-1}$. O diâmetro foi considerado igual a $4.265 \times 10^{-3} \mathrm{~m}$ e a condutividade térmica igualada à do milho, $0.315 \mathrm{~W} \mathrm{~m}^{-1}{ }^{\circ} \mathrm{C}^{-1}$ (Istadi e Sitompul, 2002) e a difusividade térmica foi calculada pela Equação 11.

$\propto_{\mathrm{S}}=\frac{\kappa_{\mathrm{S}}}{\rho_{\mathrm{S}} \mathrm{cp}_{\mathrm{S}}}$

Em que $\mathrm{cp}_{\mathrm{S}}$ é o calor específico do grão $\left(\mathrm{kJ} \mathrm{kg}^{-1}{ }^{\circ} \mathrm{C}^{-1}\right)$.

O valor do calor de dessorção do grão, $\lambda_{\mathrm{D}}$, foi igualado ao calor de vaporização da água pura, conforme Treybal (1980), Equação 12.

$\lambda_{\mathrm{D}}=2.162 \times 10^{3}+2 \mathrm{~T}_{\mathrm{S}}$

O coeficiente de difusividade efetiva, $\mathrm{D}_{\text {eff }}$, foi determinado experimentalmente por Menezes (2014), resultando nas Equações 13 a 15.

$\mathrm{D}_{\text {eff }}=5.0^{-13} \mathrm{~T}_{\mathrm{G}}-1.15^{-10}$ para $0.8 \mathrm{~m} \mathrm{~s}^{-1}(13)$

$\mathrm{D}_{\text {eff }}=6.7^{-13} \mathrm{~T}_{\mathrm{G}}-1.17^{-10}$ para $1.2 \mathrm{~m} \mathrm{~s}^{-1}(14)$

$\mathrm{D}_{\mathrm{eff}}=1.0^{-12} \mathrm{~T}_{\mathrm{G}}-1.17^{-10}$ para $1.6 \mathrm{~m} \mathrm{~s}^{-1}(15)$ 
O coeficiente de transferência convectivo de massa, $\mathrm{k}_{\mathrm{S}}$, foi estimado empregando o método de otimização nonlinearsimplex, utilizando o comando NLPSolve, sendo que o parâmetro foi determinados após minimização do erro quadrático dos desvios da umidade do leito (valor calculado e valor experimental).

\section{RESULTADOS E DISCUSSÃO}

$\mathrm{Na}$ resolução do sistema de equações diferenciais parciais, Equações 1 e 2, concomitantemente com a estimação do coeficiente de transferência de massa, $\mathrm{k}_{\mathrm{S}}$, estes variaram entre $3.32 \times 10^{-5} \quad \mathrm{e}$ $2.15 \times 10^{-4} \mathrm{~m} \mathrm{~min}^{-1}$, respectivamente para a secagem conduzida a $50{ }^{\circ} \mathrm{C}$ e $0.8 \mathrm{~m} \mathrm{~s}^{-1}$ e $80{ }^{\circ} \mathrm{C}$ e $1.6 \mathrm{~m} \mathrm{~s}^{-1}$, conforme apresentado na Tabela 1 .

Tabela 1 - Valores de $\mathrm{k}_{\mathrm{S}}$ estimados pelo modelo

\begin{tabular}{lll}
\hline \multicolumn{2}{l}{ Condição de Secagem } & $\mathrm{k}_{\mathrm{S}}(\mathrm{m} / \mathrm{min})$ \\
\hline $0,8 \mathrm{~m} / \mathrm{s}$ & $50{ }^{\circ} \mathrm{C}$ & $3,32118 \times 10^{-5}$ \\
& $60{ }^{\circ} \mathrm{C}$ & $5,27372 \times 10^{-5}$ \\
& $70^{\circ} \mathrm{C}$ & $6,43031 \times 10^{-5}$ \\
& $80^{\circ} \mathrm{C}$ & $9,20999 \times 10^{-5}$ \\
$1,2 \mathrm{~m} / \mathrm{s}$ & $50{ }^{\circ} \mathrm{C}$ & $3,27112 \times 10^{-5}$ \\
& $60{ }^{\circ} \mathrm{C}$ & $7,56268 \times 10^{-5}$ \\
& $70^{\circ} \mathrm{C}$ & $1,09462 \times 10^{-4}$ \\
& $80^{\circ} \mathrm{C}$ & $3,02218 \times 10^{-4}$ \\
$1,6 \mathrm{~m} / \mathrm{s}$ & $50^{\circ} \mathrm{C}$ & $4,11342 \times 10^{-5}$ \\
& $60{ }^{\circ} \mathrm{C}$ & $9,06367 \times 10^{-5}$ \\
& $70{ }^{\circ} \mathrm{C}$ & $2,01827 \times 10^{-4}$ \\
& $80^{\circ} \mathrm{C}$ & $2,15466 \times 10^{-4}$
\end{tabular}

Da Tabela 1, observa-se que, mantendo a velocidade de secagem constante, e aumentando a temperatura, há incremento no valor do coeficiente convectivo de transferência de massa. Os valores ajustados foram acordados com trabalhos da literatura, como Ciro-Velásquez, Abud-Cano e PérezAlegría (2010), cujos valores ajustados pelo modelo de secagem de grãos de café em camada fina, variaram entre $2.622 \times 10^{-6} \mathrm{e}$
$1.168 \times 10^{-5} \mathrm{~m} \mathrm{~min}^{-1}$, respectivamente para a secagem conduzida a $0.2 \mathrm{~m} \mathrm{~s}^{-1}$ e $40{ }^{\circ} \mathrm{C}$ e $60{ }^{\circ} \mathrm{C}$, e Silva et al. (2010), que ajustaram o valor de $2.279 \times 10^{-5} \mathrm{~m} \mathrm{~min}^{-1}$, para a secagem de grãos de arroz a $1.5 \mathrm{~m} \mathrm{~s}^{-1}$ e $60{ }^{\circ} \mathrm{C}$.

Os resultados obtidos na análise estatística são dispostos na Tabela 2.

Observa-se na Tabela 2 que, em relação ao comportamento normativo, para todos as condições testadas, o p-valor apresentou-se maior que 0.05 , indicando que a diferença existente entre os valores experimentais e os obtidos pelo modelo não foi significante. Para análise estatística desses resultados, foi calculado o erro máximo e o erro médio entre a umidade calculada e a experimental.

O maior erro médio, $\overline{\mathrm{E}}$, calculado foi de $3.3 \%$ e o maior erro máximo foi de $15 \%$, verificados respectivamente na secagem conduzida a $80^{\circ} \mathrm{C}$ e 1.2 e $0.8 \mathrm{~m} \mathrm{~s}^{-1}$, o valor de erro médio calculado indica que o modelo foi apropriado para descrever a cinética da secagem convectiva em camada fina, uma vez que ficou em torno de $2.3 \%$.

Ainda, da Tabela 2, observam-se os valores do coeficiente de determinação $\left(\mathrm{R}^{2}\right)$ para todas condições de secagem simuladas, a fim de verificar a qualidade do modelo, sendo que todos os valores foram superiores a $99 \%$, indicando boa relação entre os dados experimentais da literatura e os resultados obtidos nas simulações. Outra análise estatística que pode ser feita, para determinar se o modelo é preditivo, é o chamado teste F, que segundo Barros Neto, Scarrmínio e Bruns (1995), deve apresentar razões de Fcalculado/Ftabelado, ao nível de $95 \%$ de confiança, superiores a 5. A partir do teste F, observa-se que, em todas as condições simuladas, o modelo descreveu satisfatoriamente os casos investigados, e, portanto, o modelo desenvolvido para a secagem convectiva em camada fina pode ser utilizado para fins preditivos. 
Tabela 2 - Resultados estatísticos da simulação da secagem convectiva em camada fina

\begin{tabular}{llllllll}
\hline \multicolumn{2}{c}{ Condição de secagem } & $\begin{array}{l}\text { Erro } \\
\text { médio }\end{array}$ & $\begin{array}{l}\text { Erro } \\
\text { máximo }\end{array}$ & $\mathrm{F}$ & valor-P & F crítico & $\mathrm{R}^{2}$ \\
\hline $0.8 \mathrm{~m} \mathrm{~s}^{-1}$ & $50^{\circ} \mathrm{C}$ & 0.023929 & 0.096791 & 5963.910 & 0.912905 & 3.932437 & 0.9913 \\
& $60{ }^{\circ} \mathrm{C}$ & 0.018178 & 0.069765 & 15585.58 & 0.982513 & 3.934253 & 0.9967 \\
& $70^{\circ} \mathrm{C}$ & 0.019477 & 0.081735 & 15579.65 & 0.987952 & 3.938111 & 0.9968 \\
& $80{ }^{\circ} \mathrm{C}$ & 0.027483 & 0.155470 & 8129.523 & 0.962869 & 3.944539 & 0.9943 \\
$1.2 \mathrm{~m} \mathrm{~s}^{-1}$ & $50^{\circ} \mathrm{C}$ & 0.020767 & 0.093674 & 60196.44 & 0.971951 & 3.893640 & 0.9985 \\
& $60^{\circ} \mathrm{C}$ & 0.021251 & 0.074360 & 28288.40 & 0.963497 & 3.903366 & 0.9973 \\
& $70{ }^{\circ} \mathrm{C}$ & 0.026787 & 0.104936 & 24635.31 & 0.977186 & 3.907782 & 0.9971 \\
$1.6 \mathrm{~m} \mathrm{~s}^{-1}$ & $80^{\circ} \mathrm{C}$ & 0.033962 & 0.102887 & 47727.21 & 0.960043 & 3.909729 & 0.9985 \\
& $50^{\circ} \mathrm{C}$ & 0.026783 & 0.069104 & 42511.87 & 0.94996 & 3.894232 & 0.9979 \\
& $60{ }^{\circ} \mathrm{C}$ & 0.024680 & 0.080678 & 28062.43 & 0.964867 & 3.899502 & 0.9971 \\
& $70{ }^{\circ} \mathrm{C}$ & 0.026434 & 0.111907 & 30297.17 & 0.966144 & 3.903366 & 0.9974 \\
& $80^{\circ} \mathrm{C}$ & 0.032081 & 0.125291 & 25684.14 & 0.962814 & 3.921478 & 0.9977
\end{tabular}

Dos resultados, observou-se que a velocidade do ar de secagem não apresentou influência na cinética de secagem. Resultado semelhante foi encontrado por Barrozo et al. (2006), que estudando a secagem convectiva de grãos de soja, verificaram que velocidades diferentes resultaram em teores de umidade bastante próximo. No trabalho, os autores concluíram que, nas condições estudadas, a resistência à transferência de massa convectiva foi desprezível.

Como a velocidade do ar não apresentou efeito nas curvas de secagem, em todos os casos estudados, a resistência ao movimento da umidade na superfície dos grãos (resistência externa) pode ser considerada insignificante (Maskan et al. 2002). Dessa forma, o movimento interno da umidade (resistência interna) foi a principal resistência à taxa de perda de umidade. De acordo Geankoplis (1993), essa resistência interna é evidenciada pela presença de período de taxa decrescente de secagem, característica de grãos e sementes.

As curvas de secagem em camada fina, conduzidas a temperaturas de 50,60, 70 e $80{ }^{\circ} \mathrm{C}$ e velocidade de $0.8,1.2$ e $1.6 \mathrm{~m} \mathrm{~s}^{-1}$, são ilustradas, respectivamente, nas Figuras 2, 3 e 4.
Figura 2 - Curvas de secagem conduzida a $0.8 \mathrm{~m} \mathrm{~s}^{-1}$

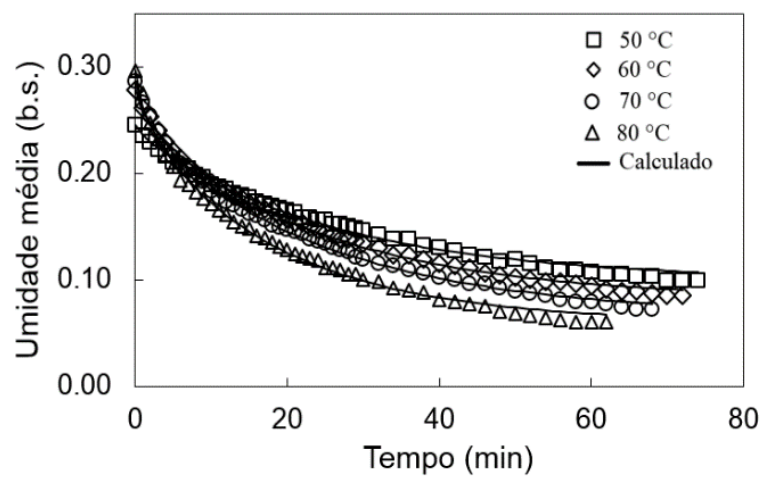

Figura 3 - Curvas de secagem conduzida a $1.2 \mathrm{~m} \mathrm{~s}^{-1}$

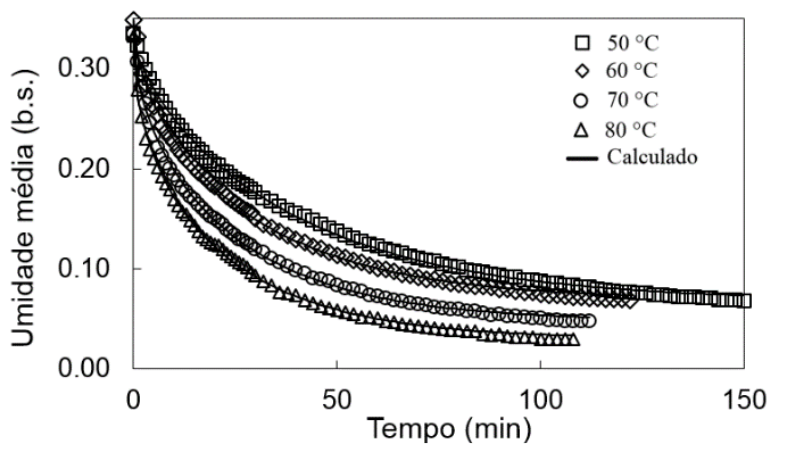


Figura 4 - Curvas de secagem conduzida a $1.6 \mathrm{~m} \mathrm{~s}^{-1}$

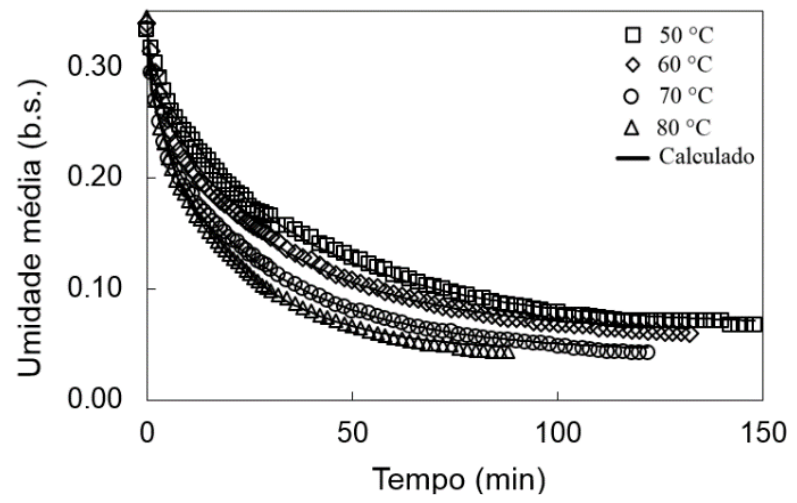

Nas Figuras 2 a 4, pode-se observar a influência da temperatura do ar na secagem convectiva. Verificou-se que, mantendo a velocidade constante e aumentando a temperatura do ar, obtém-se tempos de equilíbrio distintos. Quanto maior a temperatura, menor é o tempo necessário para se alcançar o equilíbrio entre a umidade dos grãos e o ar de secagem.

Dentre todos os dados simulados, do ponto de vista estatístico, os melhores resultados foram aqueles obtidos nas condições de secagem conduzida a $80{ }^{\circ} \mathrm{C}$ e $1.2 \mathrm{~m} \mathrm{~s}^{-1}$, com $\mathrm{R}^{2}$ de 0.9985 e relação Fcalculado/Ftabelado de 12207.29. Nessas condições, a Figura 5 apresenta as curvas de secagem dos grãos.

Figura 5 - Perfil de umidade dos grãos, para $\mathrm{o}$ ar de secagem a $1.2 \mathrm{~m} \mathrm{~s}^{-1} \mathrm{e} 80^{\circ} \mathrm{C}$

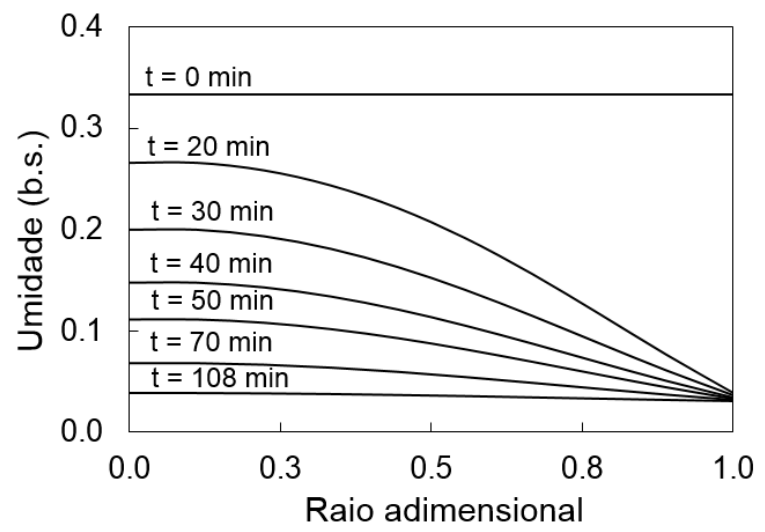

Na Figura 5, é possível identificar que a transferência de massa é reduzida a medida que a secagem atinge os estágios finais. Em relação aos raios adimensionais, os perfis de umidade dos grãos apresentam valores diferenciados a partir do raio adimensional igual a 0.20 , o que pode indicar que é a partir desse ponto que existe a resistência à transferência de massa dentro dos grãos.

A Figura 6 apresenta a comparação entre a temperatura superficial experimental, medida por Menezes (2014), e os valores calculados pelo modelo, para a secagem conduzida a $1.2 \mathrm{~m} \mathrm{~s}^{-1}$ e $80^{\circ} \mathrm{C}$.

Figura 6 - Temperatura superficial dos grãos, para o ar de secagem a $1.2 \mathrm{~m} \mathrm{~s}^{-1} \mathrm{e} 80^{\circ} \mathrm{C}$

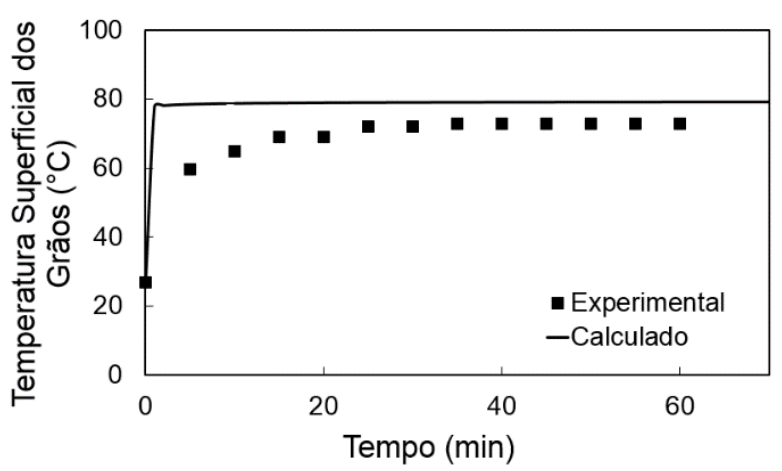

Observa-se na Figura 6, que o leito sofreu rápido aquecimento, e, em torno de 20 minutos, a temperatura da superfície dos grãos era próxima à temperatura do ar de secagem. A temperatura da superfície dos grãos apresentou-se, ao longo de todo o processo, inferior à temperatura do ar de secagem. Quando os grãos entraram em contato com o ar de secagem, estes realizaram trocas até que suas pressões de vapor e temperaturas fossem semelhantes, atingindo o equilíbrio energético, hídrico e térmico. Enquanto a pressão de vapor do ar foi menor do que a dos grãos, houve secagem, e enquanto a temperatura do ar era maior do que o dos grãos, estes sofreram aquecimento.

A Figura 7, por sua vez, apresenta o perfil de temperatura ao longo do grão, para a 
secagem conduzida nas mesmas condições da Figura 6.

Figura 7 - Perfil de temperatura no interior dos grãos, para o ar de secagem a $1.2 \mathrm{~m} \mathrm{~s}^{-1}$ e $80^{\circ} \mathrm{C}$
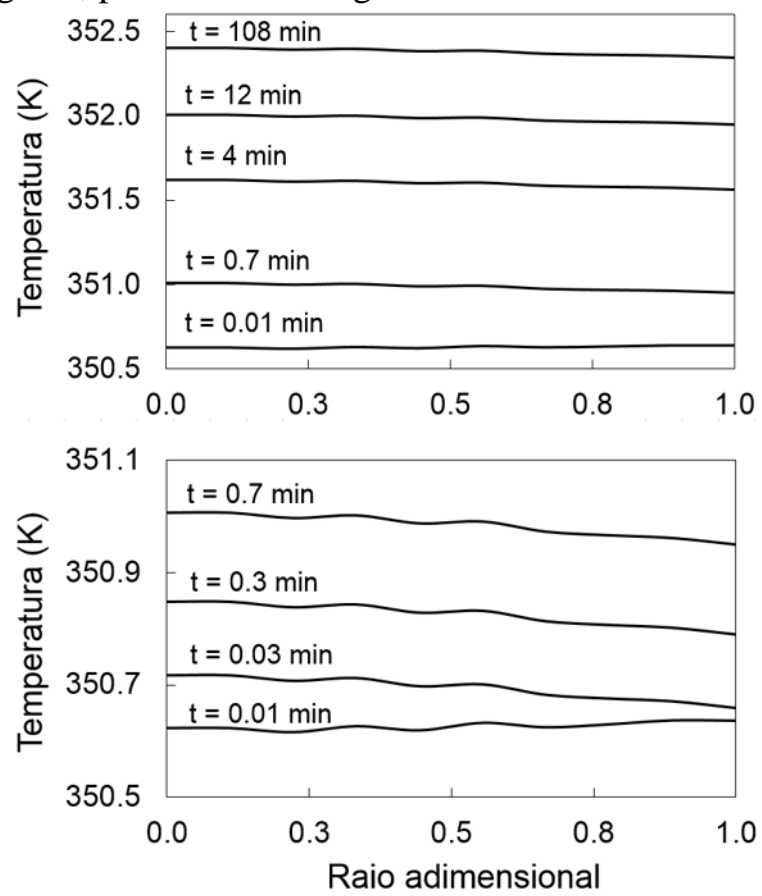

Da Figura 7, verifica-se que a temperatura dos grãos, após a rápida elevação, permaneceu constante, com pouca variação ao longo do raio. Nesse caso, quando a pressão parcial de vapor de água no ar de secagem for muito inferior e/ou a temperatura do ar de secagem for muito superior à dos grãos, a velocidade da secagem é alta. No entanto, a qualidade dos grãos e sementes pode ser afetada, uma vez que estes se tornam mais quebradiços, devido à redução da consistência interna do material, alterando sua higroscopicidade.

A análise das Figuras 5 e 7 possibilita a verificação de que o teor de umidade, cuja nitidez dos perfis diminuíram gradualmente com o tempo, variou de forma mais acentuada com o raio adimensional que a temperatura. Esse comportamento acontece em virtude do elevado número de Lewis, que pode ser entendido como a relação entre as resistências internas de transferência de massa e calor (Jumah et al. 1996).

\section{CONCLUSÕES}

Foi estudado o processo de secagem convectiva de grãos de uva em camada fina, sendo proposto um modelo matemático de parâmetros distribuídos, um método de solução das equações obtidas e validados o modelo, método de resolução e as considerações por meio de comparação com dados da literatura.

A modelagem matemática da secagem convectiva e as simplificações propostas, somadas ao método numérico utilizado para resolver o sistema de equações, foi eficiente na descrição do fenômeno de secagem convectiva de grãos de uva em camada fina, nas condições de operação simuladas. Dentre as condições de secagem simuladas, os perfis de umidade experimental e calculado, apresentaram um erro médio de $2.3 \%$ e um erro máximo de $15 \%$, este último verificado na secagem conduzida a $1.2 \mathrm{~m} \mathrm{~s}^{-1}$ e $80{ }^{\circ} \mathrm{C}$. Pela análise estatística do teste $\mathrm{F}$, ao nível de $95 \%$ de confiança, e pelo teste de $\mathrm{R}^{2}$, este último apresentando valores superiores a $99 \%$ de correlação entre os dados experimentais e os simulados, nas condições analisadas, concluiu-se que o modelo desenvolvido para a secagem convectiva de grãos de uva em camada fina pode ser utilizado para fins preditivos.

O coeficiente de transferência de massa, estimado pelo modelo variou entre $3.32 \times 10^{-5} \mathrm{e}$ $2.15 \times 10^{-4} \mathrm{~m} \mathrm{~min}^{-1}$. Nos perfis de umidade e temperatura dos grãos em diferentes tempos de secagem, foi possível identificar que a transferência de massa é reduzida a medida que a secagem atinge os estágios finais, e que, partir do raio adimensional igual a 0.20 , existe a resistência à transferência de massa dentro dos grãos.

NOMENCLATURA 


\begin{tabular}{|c|c|}
\hline & Atividade da água (-) \\
\hline & Base seca $(\mathrm{kg} / \mathrm{kg})$ \\
\hline $\mathrm{S}$ & $\begin{array}{l}\text { Calor específico do grão }\left(\mathrm{kJ} \mathrm{kg}^{-1} \mathrm{c}\right. \\
\left.{ }^{1}\right)\end{array}$ \\
\hline eff & $\begin{array}{l}\text { Coeficiente de difusividade efetiva } \\
\left(\mathrm{m}^{2} \min ^{1}\right)\end{array}$ \\
\hline & Erro médio (-) \\
\hline$c$ & $\begin{array}{l}\text { Coeficiente de transferência de calor } \\
\text { convectivo }\left(\mathrm{W} \mathrm{m}^{-1}{ }^{\circ} \mathrm{C}^{-1}\right)\end{array}$ \\
\hline s & $\begin{array}{l}\text { Coeficiente de transferência de } \\
\text { massa }\left(\mathrm{m} \mathrm{min}^{-1}\right)\end{array}$ \\
\hline 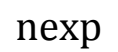 & Número de dados experimentais \\
\hline & $\operatorname{radial}(\mathrm{m})$ \\
\hline & Raic \\
\hline & das Equações 9 e $10\left(10^{5}\right.$ \\
\hline & Tem \\
\hline G & Temperatura do ar de secagem $\left({ }^{\circ} \mathrm{C}\right)$, \\
\hline $\mathrm{G}_{0}$ & $\begin{array}{l}\text { Temperatura inicial do ar de } \\
\text { secagem }\left({ }^{\circ} \mathrm{C}\right)\end{array}$ \\
\hline 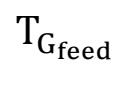 & $\begin{array}{l}\text { Temperatura de alimentação do ar } \\
\text { de secagem }\left({ }^{\circ} \mathrm{C}\right)\end{array}$ \\
\hline & do grão $\left({ }^{\circ} \mathrm{C}\right)$ \\
\hline $\mathrm{S}_{0}$ & a inicial do grão $\left({ }^{\circ} \mathrm{C}\right)$ \\
\hline 0 & Um \\
\hline 0 & Umid \\
\hline$X_{E}$ & equilíbrio (b.s.) \\
\hline EXI & erimental (b.s.) \\
\hline $\mathrm{CA}$ & Umidade calculado (b.s.) \\
\hline & agem (b.s.) \\
\hline$Y_{0}$ & Umidade ambiente do ar (b.s.) \\
\hline 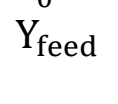 & $\begin{array}{l}\text { Umidade de alimentação do ar de } \\
\text { secagem (b.s.) }\end{array}$ \\
\hline$\propto_{\mathrm{S}}$ & $\begin{array}{l}\text { Difusividade térmica do grão } \\
\left(\mathrm{m}^{2} \min ^{-1}\right)\end{array}$ \\
\hline & Calor de dessorção $\left(\mathrm{kJ} \mathrm{kg}^{-1}\right)$ \\
\hline 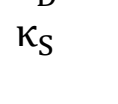 & $\begin{array}{l}\text { Condutividade térmica do } \\
\left(\mathrm{W} \mathrm{m}{ }^{-1{ }^{\circ}} \mathrm{C}^{-1}\right)\end{array}$ \\
\hline & Densidade do grão $\left(\mathrm{kg} \mathrm{m}^{-3}\right)$ \\
\hline
\end{tabular}

\section{REFERÊNCIAS}

ALONSO, D. M. J. Modeling of grain dryers: thin layer to deep beds. 2011. Dissertação (Mestrado em Bioresource
Engineering), McGill University - Montreal, 2011.

BAKER, C. G. J. Industrial Drying of Foods. Blackie Academic and Professional: London, UK, 1997.

BARROS NETO, B., SCARRMÍNIO, I. S., BRUNS, R. E. Planejamento e otimização de experimentos. Editora da UNICAMP, Campinas, 1995.

BARROZO, M. A. S., HENRIQUE, H. M., SARTORI, D. J. M., FREIRE, J. T. The use of the orthogonal collocation method on the study of the drying kinetics of soybean seeds. Journal of Stored Products Research, v.42, p. 348-356, 2006.

BROOKER, D. B., BAKKER-ARKEMA, F. W., HALL, C. W. Drying cereal grains. AVI Publishing Company, Westport, CT, USA, 1974.

CIRO-VELÁSQUEZ, H. J., ABUD-CANO, L. C., PÉREZ-ALEGRÍA, L. R. Numerical simulation of thin layer coffee drying by control volumes. Dyna, v.163, p.270-278, 2010.

DAVIDOV-PARDO, G., MCCLEMENTS, D. J. Nutraceutical delivery systems: Resveratrol encapsulation in grape seed oil nanoemulsions formed by spontaneous emulsification. Food Chemistry, v.167, p.205-212, 2015.

GEANKOPLIS, C. J. Transport Processes and Unit Operations. 3. ed. Englewood Cliffs: Prentice-Hall, 1993.

GUTIÉRREZ, L. F., RATTI, C., BELKACEMI, K. Effects of drying method on the extraction yields and quality of oils from quebec sea buckthorn (Hippophae rhamnoides L.) seeds and pulp. Food Chemistry, v.106, p.896-904, 2008. 
HELLEVANG, P. E. K. Grain drying. NDSU Extension Service, AE701, North Dakota State University, Fargo, North Dakota, 2013.

ISTADI, I., SITOMPUL, J. P. A comprehensive mathematical and numerical modeling of deep-bed grain drying. Drying Technology, v.20, p.1123-1142, 2002.

JUMAH, R. Y., MUJUMDAR, A. S., RAGHAVAN, G. S. V. A Mathematical Model for Constant and Intermittent Batch Drying of Grains in a Novel Rotating Jet Spouted Bed. Drying Technology, v.14, p. 765-802, 1996.

MAJD, K. M., KARPARVARFARD, S. H., FARAHNAKY, A., JAFARPOUR, K. Thermodynamic of Water Sorption of Grape Seed: Temperature Effect of Sorption Isotherms and Thermodynamic Characteristics. Food Biophysics, v.8, p.1-11, 2013.

MASKAN, A., KAYA, S., MASKAN, M. Hot air and sun drying of grape leather (pestil). Journal of Food Engineering, v.54, p. 81-88, 2002.

MENEZES, M. L. Estudo dos processos de secagem e extração para produção de óleo bruto de semente de uva. 2014. Tese (Doutorado em Engenharia Química) Universidade Estadual de Maringá, Maringá, 2014.

MULET, A., CÁRCEL, J. A., SANJUÁN, N., BON, J. New Food Drying Technologies - Use of Ultrasound. Food Science and Technology International, v.9, p. 215-221, 2003.

RAGHAVAN, V. G. S., SOSLE, V. Grain Drying. In A. S. Mujumdar (Ed.), Handbook of industrial drying. Boca Raton, FL: CRC/Taylor \& Francis, 2007.
ROSSELLÓ, C., SIMAL, S., SANJÚAN, N., MULET, A. Non isotropic mass transfer model for green bean drying. Journal of Agriculture and Food Chemistry, v.45, p. 337-342, 1997.

SILVA, W. P. , PRECKER, J. W. , SILVA, C. M. D. P. S., GOMES, J. P. Determination of effective diffusivity and convective mass transfer coefficient for cylindrical solids via analytical solution and inverse method: Application to the drying of rough rice. Journal of Food Engineering, v.98, p.302308, 2010.

SIMAL, S., BENEDITO, J., SÁNCHEZ, E. S., ROSSELLÓ, C. Use of ultrasound to increase mass transport rates during osmotic dehydration. Journal of Food Engineering, v.36, p. 323-336, 1998.

SIMAL, S., FEMENIA, A., GARCIAPASCUAL, P., ROSSELLÓ, C. Simulation of the drying curves of a meat-based product: effect of the external resistance to mass transfer. Journal of Food Engineering, v.58, p. 193-199, 2003.

TREYBAL, R. E. Mass-transfer operations, New York: McGraw Hill, 1980.

\section{AGRADECIMENTO}

Os autores agradecem a Coordenação de Aperfeiçoamento de Pessoal de Nível Superior (CAPES), pela concessão da bolsa de doutorado à aluna Gracielle Johann. 